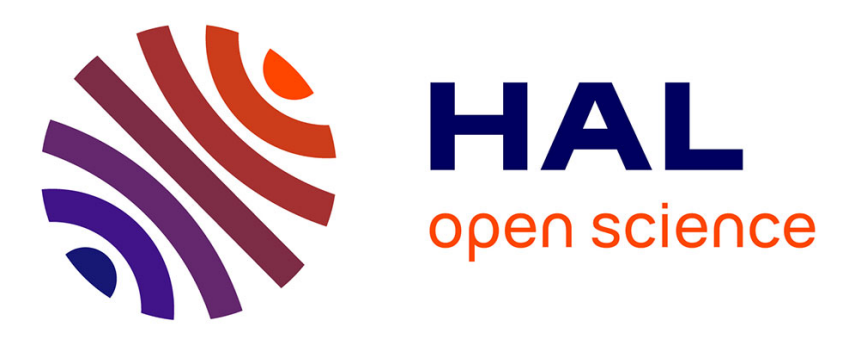

\title{
Sous les conventions. Accompagnement social à l'insertion : entre sollicitude et sollicitation
}

Marc Breviglieri, Joan Stavo-Debauge

\section{To cite this version:}

Marc Breviglieri, Joan Stavo-Debauge. Sous les conventions. Accompagnement social à l'insertion: entre sollicitude et sollicitation. Eymard-Duvernay. L'économie des conventions, méthodes et résultats. Tome II Développements, Éditions La Découverte, pp.129-144, 2006. hal-03249430

\author{
HAL Id: hal-03249430 \\ https://hal.science/hal-03249430
}

Submitted on 22 Jun 2021

HAL is a multi-disciplinary open access archive for the deposit and dissemination of scientific research documents, whether they are published or not. The documents may come from teaching and research institutions in France or abroad, or from public or private research centers.
L'archive ouverte pluridisciplinaire HAL, est destinée au dépôt et à la diffusion de documents scientifiques de niveau recherche, publiés ou non, émanant des établissements d'enseignement et de recherche français ou étrangers, des laboratoires publics ou privés. 


\title{
SOUS LES CONVENTIONS
}

\author{
ACCOMPAGNEMENT SOCIAL À L'INSERTION : ENTRE SOLLICITUDE ET \\ SOLLICITATION
}

Réf. : Breviglieri Marc \& Stavo-Debauge Joan, 2006, «Sous les conventions. Accompagnement social à l'insertion: entre sollicitude et sollicitation », in Eymard-Duvernay (dir.), L'économie des conventions. Méthodes et résultas. Tome II, Éditions La Découverte, Paris, 129-144.

brevig@ehess.fr; j.stavo-debauge@,voila.fr

Notre contribution ouvre un espace de discussion autour de la question : comment et pourquoi minorer le rôle des conventions dans l'agir commun ? Elle prend appui sur une ethnographie réalisée auprès de travailleurs sociaux [Breviglieri, Pattaroni, Stavo-Debauge, 2003 ; Breviglieri, 2005], et plus spécifiquement, croisant la problématique de l'insertion professionnelle, à propos des dispositifs institutionnels TRACE et Parrainage à l'emploi instaurés par les Missions Locales [Stavo-Debauge, 2005]. Ces dispositifs invitent particulièrement à la réflexion sur un détour hors des conventions dans la mesure où une vocation première de ceux-ci consiste à encadrer l'usager pour le former aux exigences conventionnelles du marché du travail. Or, non seulement cette formation suppose a priori un départ hors des conventions (puisqu'elle est censée y conduire), mais elle s'adresse en général à un public vulnérable qui doit être accompagné avec tact pour avoir déjà parfois connu le décrochage de divers cadres institutionnels. Cet accompagnement, tel que nous l'avons observé, peut chercher à suspendre l'horizon du jugement, à éviter les opérations de qualification, à se laisser aller un temps hors de tout cadre conventionnel, au bénéfice d'un bienfait recherché dans une relation proche et étroitement interpersonnelle.

On ne pourra établir la description de ce passage sous les conventions sans avoir au préalable montré que le registre de la convention place haut la barre des exigences capacitaires auprès des agents et sans tenir pour acquis que ce dernier ne couvre pas l'ensemble des "régimes d'engagements» dans le monde [Thévenot, 1994 et 2005]. «Éprouvantes épreuves» qui requièrent de se hisser et de se tenir au niveau exigé par les conventions : non seulement celles-ci réclament des capacités morales, cognitives et volitives significatives, mais dans leur disposition à la généralité, elles déconsidèrent un certain nombre de phénomènes singuliers et de dimensions relationnelles proximales, induisant par là un fâcheux déni de reconnaissance. C'est pourtant dans le travail de la proximité, dont les propriétés restent un point obscur des sciences sociales, qu'une part essentielle du soin, apporté au devant des vulnérabilités révélées au seuil des épreuves, s'effectue. Dans l'observation de l'activité des travailleurs sociaux, nous tenterons d'éclairer comment se distille, au carrefour de plusieurs registres d'engagement dans le rapport à autrui, une certaine pédagogie par laquelle il s'agit de soigner tout en préparant un public aux exigences de l'insertion sur un marché de l'emploi. 


\section{CE QUE PRÉSUME LA CONVENTION : LA PERSONNE MORALEMENT RESPONSABLE}

Notre point de départ consiste à nous interroger sur le type de capacités portées au premier plan dans l'usage ordinaire des conventions. Nous parlerons essentiellement de l'anthropologie capacitaire présupposée par les travaux affiliés à l'Économie des conventions. Elle implique une acception particulière de la personne dont on verra qu'elle pointe vers le pôle de la personne moralement responsable, ce qui alimente par de nouveaux enjeux une science économique classiquement tournée vers le pôle de l'acteur rationnel.

Un premier plan d'exigences capacitaires se rapporte directement à la convention en soi. La première mise de fond réclamée par la nature de la convention consiste en une minimale motion volontaire orientée vers un agir ensemble, une volonté de coopérer. Dans cette énonciation d'une intention d'action [Livet, 1993], quelque soit son degré de lisibilité, se loge une promesse. Précisément une promesse à s'engager dans un "agir ensemble » à venir. En cela, dans le répertoire des réquisits capacitaires pointe une exigence morale conséquente qui correspond à une disponibilité maintenue appelée par l' "agir ensemble » et adossée à la durabilité et à l'assurance offertes par la promesse.

Un second plan d'exigences capacitaires naît de la coexistence de plusieurs dispositifs conventionnels répartis sur des axes de valeur différentiables. C'est sur ce point important que l'Économie des conventions fraye un domaine de réflexion sur des cadres complexes de coordination, notamment relatifs à des modes de transaction hors marché. Mais elle ouvre aussi une perspective sur les capacités pragmatiques des acteurs. Tout d'abord, sur des capacités au jugement ordinaire qui entraînent des aptitudes relatives à un agir circonspect (apprécier, estimer, évaluer, interpréter) prédisposant la personne à l'enquête et à la critique. De plus, parce que le jugement s'appuie aussi sur des opérations de rapprochement et de qualification permises par des principes d'équivalence, une aptitude à la généralisation prédispose la personne aux rapports en public [Boltanski \& Thévenot, 1991]. Enfin, étant donné l'existence d'une pluralité des formes de justice, démultipliant le caractère des attentes conventionnelles et des qualifications portant sur le monde, la personne démontre une véritable " plasticité » en changeant plus ou moins régulièrement d'état [Dodier, 1991]. Cette «plasticité » repose sur un pouvoir supposé du jugement critique, associé à des capacités remarquables de révision et d'ajustement de l'action. Aux limites de la connaissance et de la coordination pointées par le jugement critique se dévoile une disposition essentielle à l'apprentissage en situation ${ }^{1}$. La nature essentielle de cette disposition dépend en réalité du statut éminemment conséquent de l'erreur envisagée du seul point de vue des attentes conventionnelles. Elle ne correspond pas seulement à la mise en échec de l'action, mais dispose d'emblée du statut moral de faute: elle compromet pour un temps ou de manière irréparable la solidarité et la félicité de l'action commune.

Finalement, la mise ensemble de plusieurs dispositifs conventionnels laisse entrevoir les efforts nécessaires pour que les individus puissent s'accorder sur un sens de la réalité des figures du bien engagées dans une action en commun [Thévenot, 2005]. Aussi, si cela nous éloigne d'un modèle du vivre ensemble unique car fondé sur l'équilibre spontané résultant de choix rationnels, la question de l'engagement en appelle à une autre économie. En effet, tout engagement est une responsabilité et soulève une question morale relative au « vouloir vivreensemble » dont l'approche conventionnaliste envisage, à sa manière, la pluralité des formes. C'est donc d'une économie de la responsabilité dont il est question, reposant sur une

\footnotetext{
${ }^{1}$ Disposition qui se trouve au pôle opposé de la «disposition sociale » ou de l'habitus dont l'acquisition est une affaire d'héritage et d'intériorisation de schémas pratiques.
} 
anthropologie exigeante en capacités morales puisqu'elle réclame que l'individu se rende comptable à différents stades de son action, des attendus de celle-ci comme de ses conséquences $^{2}$.

\section{LES EXIGENCES CAPACITAIRES DANS L'HORIZON DU CONTRAT (QU'OUVRE LA CONVENTION) : TRAVAIL SOCIAL ET VISÉE D'AUTONOMIE}

\section{Le contrat et les réquisits de l'autonomie en acte}

En évoquant désormais la figure du contrat, notamment comme instrument des politiques socio-économiques d'insertion, nous allons montrer que nous ne pouvons plus contenir l'anthropologie capacitaire dans les limites supposées par un modèle de coordination fondé simplement sur des attentes conventionnelles. En un sens, dans son rapport à la convention, le contrat ne fait qu'ajouter des exigences capacitaires supplémentaires. La convention peut d'ailleurs s'envisager comme «ce qui rend possible des relations contractuelles et les garantit » [Quéré, 1993]. De son côté, l'équipement du contrat représente aussi " une garantie contre les litiges sur les conventions » [Livet, 1993]. Mais ajoutons qu'il tire en deux sens vers les principes de justice à la source des axes conventionnels. D'un côté le contrat place le rapport à autrui sous l'idée double d'un engagement à tenir et d'une conformité à la loi ; de l'autre, il entend inciter les individus à engendrer d'eux-mêmes une interprétation et une observation de critères reflétant le juste.

On retrouve bien, à travers l'objectif d'une propédeutique de l'autonomie intégré au dispositif des contrats d'insertion, cette double dimension : le contrat ne se contente jamais de faire plier la morale du côté déontologique car il se trouve également rapporté à un pôle téléologique en ce qu'il doit inciter à l'autonomie plutôt que de seulement la gouverner. Ainsi, dans son parcours d'autonomisation, l'individu en difficulté doit déjà pouvoir concilier des capacités de différents ordres (interprétatives, volitives et éthiques) afin d'assurer une coordination fondée sur le contrat. C'est ainsi au prix fort d'une démarche assumée et d'une obéissance vigilante à l'endroit du contrat qu'il est censé réaliser une percée vers l'autonomie entendue comme une participation à sa propre émancipation visant sa liberté civile.

\section{Le projet et l'extension des attendus conventionnels qu'il suppose}

Concernant les institutions du travail social, la priorité assignée à la lutte contre l'exclusion a induit le renforcement des structures locales et notamment du tissu associatif. La gravité de l'exclusion s'est jaugée à l'aune de l'épuisement des ressources de l'individu nécessaire au déploiement de ses capacités à l'autonomie. Est apparue une figure majeure de "victime » dont la vulnérabilité se reconnaît dans la perte ou la menace de l'autonomie individuelle [Ricoeur, 1991]. Sur le front de l'intervention sociale, les travailleurs sociaux, occupés par l'enjeu d'une responsabilisation des patients en vue de leur futur, ont pu jouer essentiellement de deux outils de particularisation de la relation: le contrat et le projet individuel. La

\footnotetext{
${ }^{2}$ Nous marquons ici notre dette à l'égard de la thèse de doctorat de L. Pattaroni [Pattaroni, 2005]. Des développements plus conséquents sur le sujet abordé y sont effectués.
} 
prévalence accordée à l'habillage contractualiste affirme un certain compromis pour le bien commun qui reflète l'évolution des formes légitimes de justification des politiques publiques française depuis l'après guerre. D'un côté la double matrice juridique et marchande du contrat, de l'autre côté, la double matrice industrielle et connexionniste du projet (que le contrat va tendre à inclure), se confondent pour viser des interventions locales conformes aux objectifs macro économiques de lutte contre l'exclusion ${ }^{3}$.

Ainsi, les patients de ces politiques publiques vont devoir témoigner des qualités requises pour pouvoir contractualiser, formaliser et soutenir dans la durée, un projet délibéré jusqu'aux épreuves afférentes où se met en jeu leur insertion. Aux exigences capacitaires propres du contrat (couvrant les domaines du choix, de la négociation, de la promesse) s'ajoutent les réquisits du projet (affirmation d'une action intentionnelle propre, maintien des capacités engagées sur sa durée, affranchissement progressif des entraves à sa réalisation et livraison de celui-ci devant des tiers pertinents). Un projet dont on comprend mieux la dimension fortement publique si l'on garde présent à l'esprit qu'il est instaurateur d'un avenir soumis à l'évaluation et à l'intervention des acteurs publics du travail social, mais aussi à celles d'autres acteurs privés ou publics, notamment des « intermédiaires du marché du travail» [Bessy \& Eymard-Duvernay, 1995] ainsi que des recruteurs.

\section{LA VULNÉRABILITÉ DES USAGERS AU REGARD DES ATTENTES CONVENTIONNELLES ET DANS LA PERSPECTIVE DES ÉPREUVES DE RECRUTEMENT}

\section{La faillibilité des engagements et son complexe de vulnérabilités}

En faisant culminer ces exigences capacitaires au niveau du contrat-projet, nous nous préparons à rendre visible un écueil profond des politiques publiques contractuelles centrées sur la question de l'insertion professionnelle. Pratiquement, le travailleur social se trouve dans la difficulté, parfois extrême, de concilier une prétention à l'autonomie de la part de l'usager et la reconnaissance de sa vulnérabilité. Il s'efforce d'exiger de son patient les capacités considérables dont nous avons parlé tout en exerçant sur lui une attention, particulièrement légitime, à sa fragilité. Nous verrons que pour concilier ces aspirations éloignées, l'intervenant social doit bien souvent se dégager partiellement des attentes conventionnelles pertinentes au plan des institutions sociales, passer en quelque façon sous les conventions. C'est donc en désignant d'abord les fragilités de l'usager qui minent la bonne réalisation du contrat-projet que nous ferons émerger ce nécessaire dégagement des professionnels hors du cadre conventionnel impliqué par ce dispositif.

Pour aborder ce point, et ouvrir au plus large le spectre des vulnérabilités pouvant affecter la personne, il nous faut introduire l'idée d'une pluralité de modes d'engagement dans la relation au monde. À un engagement public, dont nous avons vu qu'il se pluralise au regard d'une diversité de principes de justice, s'ajoutent d'autres modalités d'appréhension et de

\footnotetext{
${ }^{3}$ Dans une grammaire politique de type connexionniste, le projet reste l'outil idéal pour se défaire de liens préjudiciables et s'engager dans un nouveau réseau de relations; dans une grammaire politique de type industrielle, l'objectivation permise par le projet participe aux méthodes prévisionnelles recherchées pour rationaliser efficacement des activités futures [Bolanski \& Thévenot, 1991 ; Boltanski \& Chiapello, 1999].
} 
traitement de l'environnement humain et non humain ${ }^{4}$. Chaque régime d'engagement se défini comme le lieu d'articulation entre un sens de la réalité et un sens du bien [Thévenot, 2005] : un régime de l'action en public, rapporté au niveau du conventionnel et à la dimension d'un bien commun se distingue d'un régime de l'action en plan, relié au registre de la norme et à la dimension d'un bien fonctionnel, puis d'un régime de l'action habituée, induisant un rapport personnel au monde habité et s'ouvrant sur le bienfait du familier. À ces trois états de l'action, pesant différemment sur les différents canaux de coordination avec autrui, s'articulent différents visages de la personne (comme sujet des grammaires publiques, individu et proche) qui laissent à leur tour entrevoir plusieurs figures de la vulnérabilité. Ces figures concernent à la fois les régimes en propre (à chaque régime correspondent des failles particulières), et la composition même de ces régimes (composition plus ou moins heureuse au sens où c'est depuis elle que se conçoit le noyau de "consistance » de toute personne [Ibid.]).

\section{$\underline{\text { Rigidité du caractère, promesse non tenue et figuration défaillante }}$}

Les sites où s'établit le travail social relatif à l'insertion, comme les Missions Locales sur lesquelles nous reviendrons sous peu, sont naturellement soumis au devoir de renforcer la consistance des personnes et de composer avec elles sur des registres d'action tres divers. Ils s'opposent en cela au domaine du recrutement professionnel qui configure déjà une épreuve publique orientée autour d'une pluralité de critères puisant dans des "conventions de compétences » auxquelles s'ordonne l'évaluation de «qualités» [Eymard-Duvernay \& Marchal, 1997]. Le travail des intervenants sociaux consiste en partie à repérer la diversité des failles, y compris sur le registre du proche, et pas seulement comme un défaut de ressources, pour appréhender au mieux l'épreuve du public dont nous avons déjà cerné l'ampleur des exigences capacitaires.

Les faillibilités s'étendent au long de trois axes majeurs. Le premier se dit comme incapacité pour l'usager à composer les régimes d'engagements. Cet axe peut se décliner d'abord comme repli sur un caractère rigidifié. C'est à un défaut d'apprentissage que ramène finalement cette vulnérabilité du patient. Elle reflète diversement une obstination à conserver des plis cognitifs ou affectifs et une absence de souplesse dans le parcours d'insertion, bref, une prédisposition, jugée néfaste, à l'immobilité. Tout repli sur le pôle rigide du caractère indique une faille de l'usager pour le travailleur social, identifiable par des attitudes caractéristiques rapportées au " blocage » du caractère. Ainsi se distingueront un attachement viscéral du patient à ses proches (famille ou amis) qui l'empêche de se présenter «en personne » au guichet, d'un déploiement systématique de comportements calculateurs utilitaristes dans le rapport à l'institution, où enfin de l'inflexibilité d'une conviction ou de l'immobilisme dans un registre unique de grandeur sociale ${ }^{5}$.

Le second axe de faillibilité révèle des incapacités à tenir la promesse des engagements supposés par l'outillage du contrat-projet. À son niveau le plus élémentaire, l'incapacité consiste en l'absence d'un maintien de soi depuis lequel s'élève et se soutient une volonté

\footnotetext{
${ }^{4} \mathrm{Au}$ total, la pluralité « verticale » des régimes d'engagement (allant du proche au public) s'ajoute à la pluralité « horizontale » des sens du juste.

${ }^{5}$ Sur ce dernier pôle du caractère, les travailleurs sociaux témoignent majoritairement de la double hantise d'une conviction religieuse associée à un fondamentalisme et de la «grandeur domestique» du «caïd» ou du « meneur de bande » imposés et maintenus en l'état au guichet du service social. Leurs tentatives pour faire plier cette rigidité sont envisagées ultérieurement. Le cas de jeunes femmes issues de l'immigration et participant au programme TRACE est envisagé dans [Benlemselmi, 2002].
} 
[Breviglieri, 2005]. Mais elle concerne l'inconsistance de la volonté à tous les étages et à toutes les étapes de son déploiement, allant de l'embarras du choix, survenant dès lors qu'il est demandé à l'usager de se résoudre à se doter d'un projet, jusqu'à la difficulté de celui-ci à tenir parole et à le réaliser sans faillir ${ }^{6}$. Cet axe regarde ainsi la charge du transport temporel du projet et sa nécessaire résistance aux épreuves traversées. C'est en effet souvent au terme d'une accumulation d'issues négatives que se trouve atteinte la confiance que l'usager met dans ses pouvoirs. À l'épuisement des efforts mis dans le port du projet, où pointe l'abîme du découragement et de la résignation, correspond un affaissement de la volonté par laquelle tenait la promesse des engagements.

Enfin, sur un troisième axe s'alignent des faillibilités relatives à l'exposition de soi présentée sur le marché du travail. Les capacités à répondre aux attendus conventionnels sont ici inspectées dans leur mise en visibilité. Il n'est en effet plus simplement question de reconnaître la constitution de ces capacités, mais plutôt leur mise en valeur. Les faillibilités ici en jeu paraissent comme ce qui entrave une bonne présentation de l'usager aux épreuves de recrutement, gâte son heureuse figuration devant celles-ci ou bien pèse sur la félicité de la livraison publique du projet qui est le sien.

Parce que bon nombre des usagers qui viennent à la Mission Locale semblent affectés par l'une ou l'autre de ces faillibilités, les travailleurs sociaux se doivent alors de passer sous les conventions et d'abaisser d'un cran des exigences qui, pour les patients de l'action publique, se présentent de prime abord comme autant d'épreuves insurmontables ou intimidantes.

\section{LE NÉCESSAIRE PASSAGE SOUS LES CONVENTIONS}

\section{Travail de proximité et travail de la proximité}

Occupons nous désormais à éclaircir ce passage hors des bornes conventionnelles, tout en nous retournant sur l'exemple donné par les mesures d'accompagnement à l'insertion. Il faut pour cela nous montrer sensible au travail de la proximité opéré par les travailleurs sociaux et son mouvement constitutif de rapprochement où les personnes inclinent à délaisser un rapport comptable entre elles [Breviglieri, 2005]. La proximité n'est pas ici un simple point physique de contact, elle reflète plutôt une manière d'appréhender familièrement le monde [Thévenot, 1994].

Une première source de confusion se rend immédiatement sensible dans la mesure où l'on serait tenté de réduire ce travail de la proximité au «travail de proximité » aujourd'hui politiquement valorisé dans le vaste mouvement de modernisation des services publics français. Tout en en constituant pourtant un aspect, elle se distingue du simple travail de tissage de liens en réseaux et de prise en compte de la particularité des besoins d'usagers devenus des clients. Ces deux registres d'action du travail social ne se trouvent pas sous les conventions car ils déplacent des attentes conventionnelles reposant sur des principes de justice, connexionnistes d'un côté et marchands de l'autre. Ils se présentent toutefois comme des axes de justification vers lesquels le travail de la proximité, en se voyant soumis à l'évaluation, pourra aisément s'orienter ou être rabattu, par un réductionnisme et au mépris de son économie propre. Cette économie, envisagée dans le cas de la relation d'aide

\footnotetext{
${ }^{6}$ La dotation d'un projet doit s'affirmer au devant d'un tiers, travailleur social et souvent aussi collectif d'usager.
} 
personnalisée à l'insertion, repose sur l'existence de savoir-faire relatifs à l'accompagnement et la sollicitude. Ces derniers pointent vers le maintien d'une éthique de l'accueil qui considère, et par là protège, les dimensions familières attachées à la personne.

\section{La proximité familière et son écart à l'interaction comme au régime de l'Agapè}

Il importe de dissocier ces savoir-faire des compétences à l'interaction comme des dispositions au rapport compassionnel. La sollicitude dont témoigne le travail de la proximité ne peut être d'ailleurs placée sous le registre descriptif de la sociologie de l' "interaction » (d'inspiration Goffmanienne), ni de celui de la compassion dans sa figure dérivée de l'épiphanie du visage au sens de Lévinas ou dans celle de l'Agapè [Boltanski, 1990].

L'égard dans la sollicitude qui porte au rapprochement n'a pas d'exigence supérieure de réciprocité et laisse ouverte la possibilité d'un lien interpersonnel trouvant convenance dans l'asymétrie. Il place en retrait la présomption d'égalité inhérente à la co-présence supposée par l'interaction. La sociologie des interactions présuppose des " individus comme unités » [Goffman, 1973] qui, de part et d'autre, campent des entités autonomes et décisionnelles s'efforçant de trouver un équilibre interactionnel. Cet équilibre repose essentiellement sur une entreprise de négociations et " d'arrangements à toutes fins utiles » [Joseph, 1998], réclamant un important travail réciproque de figuration. Dans la perspective de recueillir les vulnérabilités de l'usager, le travailleur social peut se montrer critique devant celui qui tente de gérer la situation pour garder la face, et qui, selon lui, cherche absolument à maitriser en surface ses émotions. Inversement, le rapprochement s'effectue parfois à l'aide d'encouragements à se livrer davantage, se laisser aller à la confidence ou ne pas s'offenser pour un rien.

La dynamique du rapprochement de l'usager, qui permet le passage sous les conventions, ne recouvre pas non plus celle du « régime d'Agapè » (Boltanski, 1990). En premier lieu, parce que les intervenants sociaux prennent une distance déontologique relativement au pôle de la compassion ou à celui de la pitié. Cette distance doit être considérée sur la base d'une forte hantise de l'assistancialisme et/ou du paternalisme, soit deux formes qui mettent en demeure l'usager dans un état de passivité et dont il importe aux travailleurs sociaux de conjurer les méfaits afin de ne pas assombrir l'horizon d'une autonomisation attendue. Pour comprendre ce qui distingue une pratique de la sollicitude d'une politique de la compassion, il convient de dissocier le proche au sens du familier, paraissant à mesure du rapprochement, du proche au sens du prochain. Pour advenir, le proche comme familier suppose que des accommodements progressifs et attachants soient trouvés dans une expérience conjointe durable. La familiarité repose alors sur le socle d'une mémoire et d'une confiance conçues et éprouvées durant ce rapport intime dont l'inévitable sinuosité fait toute la singularité. $A$ contrario, le proche est un prochain en tant qu'il est habité par un sens eschatologique qui le dépasse. Le prochain appelle à l'événement ponctuel de la rencontre, à une proximité immédiate où s'initie un geste qui, bien que réfractaire à la comparaison, s'étend par une opération qui transcende sa contextualité propre jusqu'à configurer une "communauté fraternelle ».

Si le mouvement de rapprochement accompli sous les conventions cherche donc à se tenir à l'écart de la compassion qui menace de grever l'éclosion de l'autonomie de l'usager, le travail de la proximité s'inscrit de fait dans un autre registre éthique qui suppose des formes expressives et donc des aptitudes professionnelles différentes de celles établies sous le régime d'Agapè. Commandé par la faillibilité initiale de certains usagers devant des épreuves 
publiques qui charrient leur lot d'attentes conventionnelles, le rapprochement vient répondre à la nécessité momentanée de maintenir l'usager dans le cadre d'un dispositif d'action publique et de l'empêcher de retomber hors des domaines couverts par l'institution. Il est ainsi transitoire, et permet de ménager aux usagers en difficulté un accueil et de réarmer leur confiance. Une part essentielle de l'activité et de la compétence des travailleurs sociaux consiste, par la suite, à savoir prendre appui sur les bienfaits de ce rapprochement de sorte à apprêter les usagers aux épreuves de l'insertion ou bien, lorsque cela est nécessaire, afin d'atténuer la sévère morsure des évaluations rencontrées au contact ou à l'issue desdites épreuves. Comme on va le voir, le travail de la proximité mis en œuvre dans les Missions Locales, au travers de dispositifs comme TRACE ou le Parrainage à l'emploi, peut aussi se comprendre comme formation aux épreuves conventionnelles. Ces lieux figurent des « intermédiaires du marché du travail» bien spécifiques dont l'objet est parfois moins de mettre en lien des acteurs économiques que de faire franchir à certains un seuil par lequel s'assument les difficiles épreuves de l'insertion? ${ }^{7}$.

\section{LES POLITIQUES DE PROXIMITÉ COMME FORMATION AUX ÉPREUVES CONVENTIONNELLES ET COMME PÉDAGOGIE DE LA PRÉSENTATION DE SOI}

Si l'on veut atteindre la question essentielle de la formation des usagers aux épreuves de l'insertion, encore nous faut-il passer par une discussion sur la variété d'aptitudes des travailleurs sociaux, dont la faculté à passer sous les conventions est une des pièces maîtresse. Ces aptitudes touchent à plusieurs dimensions qui se conjoignent pour instaurer une dynamique de reconnaissance nécessaire à la consolidation d'une volonté requise pour se présenter aux épreuves du marché de l'emploi. Nous allons décomposer une série de niveaux où se joue une reconnaissance : du premier au troisième niveau, la reconnaissance incline à se porter davantage vers le pôle de la personne moralement responsable dont nous avons vu qu'il correspond aux exigences capacitaires propre au registre de la convention. À chaque niveau, le passage sous les conventions et le rapprochement de l'usager engagera un dispositif particulier d'attentions compétentes où s'allient sollicitude et sollicitation pour travailler à la consistance de sa personne. Ce sont ces compétences qui pourront permettre l'assise pérenne et ample d'une confiance (envers soi et l'institution) par laquelle se rend possible l'établissement du contrat-projet.

\section{L'horizon d'une constance approuvée}

Le premier étage se donne l'horizon de reconnaissance d'une constance approuvée se distribuant diversement selon que l'on considère l'une ou l'autre des personnes impliquées par l'accompagnement. D'abord, comme approbation, donnée par l'usager, à l'engrènement d'un rapport de proximité durable avec un travailleur social ${ }^{8}$. Ensuite, comme reconnaissance

\footnotetext{
${ }^{7}$ Acteurs menacés d'exclusion du fait d'une absence de ressources économiques (TRACE) ou encore arrêtés aux seuils des épreuves de recrutement du fait de l'exercice de discriminations ethniques et/ou raciales ou de la méfiance des recruteurs et des employeurs (Parrainage à l'emploi).

${ }^{8}$ Les deux dispositifs, TRACE et le Parrainage à l'emploi, activent des accompagnements personnalisés de longue durée. Nous prendrons surtout appui sur l'exemple des Parrains dont le travail de rapprochement est plus rarement entrevu dans la littérature. Durant les appariements parrain/filleul, les responsables du dispositif font en
} 
des possibilités professionnelles entrevues chez l'usager, reconnaissance qui devra s'établir dans un temps suffisamment long pour les laisser paraître comme constantes.

Les agents des Missions locales ont trouvé en la personne du Parrain un être susceptible d'amoindrir la défiance que leur public naturel («les jeunes ») ne manquait pas de témoigner contre les "représentants» d'une «institution» suspectés d'œuvrer pour un «système injuste $»^{9}$. Les propriétés du Parrain - un bénévole, non attaché à l'institution, assis sur une expérience du marché du travail local et détaché auprès d'un seul usager - permettent de désarmer la méfiance de certains usagers et d'offrir une sollicitude qui se laisse moins aisément barrer et plus facilement reconnaître. Parce qu'il n'est ni pleinement «attaché à l'institution » ni rétribué par elle, certains usagers s'autorisent la suspension de leur «humeur anti-institutionnelle ».

Si les agents de la Mission Locale sont si sensibles à la venue du parrain, c'est aussi que s'ouvre pour l'usager l'amorce d'une reprise de confiance en lui-même jugée nécessaire à la constance de ses engagements futurs. Ecoutons ce que nous dit la responsable du dispositif à propos des effets sur l'usager de l'arrivée du parrain : "Il a un petit coup de fouet en se disant "y'a quelqu'un qui vient de l'extérieur, qui n'est pas payé, qui vient donner son temps pour moi », parfois ça les étonne hein. Quand on leur parle du parrainage c'est : " mais pourquoi est-ce qu'il vient s'occuper de moi ? " ". Il importe de considérer la dimension de surprise face à une personne qui, sans mandat, s'avance et dévoile une attention inédite par laquelle l'usager reconnaît qu'il vaut considération, écoute et effort. Et comme le résume autant que l'espère la responsable du parrainage à l'emploi, le « jeune », une fois qu'il a encaissé le choc de cette surprise, a de grandes chances de se rendre à un questionnement susceptible de donner lieu à un sentiment plus stable relatif à sa valeur.

Mais l'effet positif de surprise produit par l'attention bienveillante du Parrain n'est pas en soi suffisant pour pouvoir entièrement porter le jeune aux épreuves et faire en sorte qu'il puisse résister à leurs défaveurs. Encore faut-il que le rapport s'inscrive dans une durée à travers laquelle s'établira le travail de la proximité. L'épanouissement de cette confiance accordée, inaugurant et soutenant le mouvement d'une remise de soi aux ambivalents pouvoirs d'autrui [Baier, 1986], est suspendu à l'observation, par l'usager, de la constance et de la bienveillance de l'attention que nourrit l'agent public à son égard. La fidélité d'une présence vigile et d'une disponibilité attentive s'attestera dans un travail fiable de conseils prodigués dans le cadre de l'accompagnement et animés par le moteur d'un souci relatif à son avenir personnel. La mesure d'accompagnement tire vers une politique de la reconnaissance en laissant paraitre cette approbation valant comme encouragement ; comme le dit un Conseiller Emploi, le parrain vaut d'autant plus comme un «support» et un «encouragement» que le "jeune» peut nourrir le "sentiment qu'il n'est là que pour (l)'aider ». Mais, tout l'art de l'accompagnement et de l'apprêt aux épreuves consistera à renforcer continûment le pôle de la confiance en soi de sorte à ce que cette volonté naissante puisse se déterminer, à terme, dans une autonomie.

\footnotetext{
sorte que le premier puisse valoir auprès du «jeune» comme le vivant témoignage d'une possibilité de futur le concernant (il dispose d'une situation convoitée par lui, mais il peut aussi être affecté des mêmes attributs malvenus dans le monde du travail, par exemple une origine commune passible d'un traitement discriminatoire), cela afin de lui donner espoir en lui faisant entrevoir l'ouverture d'un avenir.

${ }^{9}$ Les passages en italique et entre guillemets indiquent des citations ou des expressions communes formulées par les usagers ou les intervenants eux-mêmes.
} 


\section{L'horizon d'une composition affirmée}

Un second niveau de reconnaissance délivré dans le travail d'accompagnement de l'intervenant social s'opère sur fond d'une composition d'engagements affirmés par l'usager. Il n'y va plus simplement de possibilités reconnues à l'usager mais de capacités à composer, sans désorganisation personnelle, des desseins professionnels avec des vues ou des ambitions concernant d'autres aspects de sa personnalité, y compris dans un registre familial ou amical. Les intervenants sociaux s'assurent, à ce niveau, de rendre une souplesse au pôle identitaire du jeune et une consistance à ses engagements envers le proche comme en public. Cet horizon d'une composition affirmée non seulement donne au travailleur social des garanties face au risque que le patient de l'action public se replie sur un caractère rigidifié (dont on a vu plus haut l'enjeu en termes de faillibilité), mais il oriente l'usager dans un mouvement d'affirmation de ses projets, lestant de cette façon sa volonté d'insertion.

C'est au moyen d'entretiens avec le Conseiller Emploi, s'attachant à la biographie et à l'ordinaire des jours de l'usager, que se repèrent les éléments dispositionnels (habitudes, traits de caractères, qualités personnelles) et les moteurs vocationnels (aspirations, désirs stables, etc.) susceptibles d'étayer l'assemblage d'un projet qu'il pourra alors plus facilement dire et faire sien. Cette lente montée à la parole d'un « soi » trouvant l'occasion de se raconter dans le milieu d'une écoute attentive importe en ce qu'elle élève la personne de l'usager au niveau d'une estime et qu'elle crédibilise graduellement ses capacités propres. Il est attendu qu'à l'issue de ces entretiens l'usager puisse surmonter son sentiment initial d'impuissance et se tenir pour un être proprement individué car doté d'un caractère riche de dispositions déjà possédées et jusqu'ici continûment exercées.

Si l'on emprunte ici un langage dispositionnel, c'est que les dispositions figurent ce qui est, tout à la fois, propre, disponible, stable et permanent et dans le même temps relativement inaperçu de celui qui les porte puisqu'elles se déploieraient comme autant d'habitudes contractées qu'une réflexion peine à ressaisir sans l'aide d'un tiers - tiers qui est ici le Conseiller Emploi. En outre, celles-ci sont congruentes avec la saisie de l'identité de la personne en tant que caractère, modalité de figuration de l'identité personnelle indexée au pôle de la mêmeté par lequel il convient de passer avant que ne puisse se constituer le pôle de l'ipséité (lieu de la promesse) [Ricœur, 2004, p. 142]. Ces dispositions se prêtent ainsi à une évaluation qui donne un encouragement pour peu qu'elles se laissent reconnaître, d'abord par le Conseiller Emploi puis par l'usager lui-même, comme autant de promesses d'un futur s'offrant comme une base de capacités élémentaires avec lesquelles l'un et l'autre peuvent compter.

"Mais ça [la pratique d'un sport] pour une entreprise c'est un gage de sociabilité : pouvoir travailler avec des gens qu'on déteste, se contraindre soi-même à arriver à l'heure. "Rien ne t'oblige vraiment à arriver à l'heure pour ton entraînement, pourtant t'es à l'heure, rien ne t'oblige à arriver à l'heure au match et pourtant tu es à l'heure, le seul qui est puni si tu n'y vas pas c'est toi (...). Et tout ça pour un employeur ça peut avoir de la valeur» (...). Et parfois on parle dix minutes avec un jeune et il commence là à voir des qualités, alors que pour lui c'est un truc où il s'éclate, (...) il voit des choses qui vont lui permettre dans un entretien de parler de lui. (...) Et tout ça, c'est une manière de préparer un peu l'entretien, et de leur donner un peu confiance en eux-même, parce que le problème pour beaucoup c'est comment je vais faire pendant un quart d'heure à vingt minutes pour parler de moi. » 


\section{L'horizon d'une consistance attestée}

Comme on l'entend dans cet extrait, c'est sur une base dégagée dans des conversations que le Conseiller Emploi fait ensuite porter un travail de mise en valeur. Avérées et reconnues par l'usager, les dispositions s'élèvent au rang de compétences décisives pour se présenter et participer aux épreuves de l'insertion. Ainsi s'annonce un stade ultime de reconnaissance qui quête l'horizon d'une consistance attestée en regard du niveau institutionnel et conventionnel où se joue le recrutement. Viennent alors des moments de mise à l'épreuve où les travailleurs sociaux, en recourrant à divers dispositifs pédagogiques mêlant réalisme de l'évaluation et caractère désinhibant de la fiction du jeu, cherchent à s'assurer de la teneur et de la tenue des usagers (i.e. de leur consistance). Ils s'efforcent aussi de mesurer leur faculté à se mettre en valeur et à pouvoir faire la différence face aux recruteurs en présentant des qualités remarquables et publiquement estimables.

Alors qu'initialement, dans les premières expérimentations associatives du parrainage à l'emploi, le Parrain se devait d'introduire le jeune auprès des employeurs (cela afin de faire transiter une confiance de proche en proche et de gager l'accueil du jeune par la réputation du parrain), les travailleurs sociaux qui chapeautent maintenant le dispositif sont plus que réservés quant à cette ancienne forme d'accompagnement. La présence du parrain où se tient l'épreuve de recrutement signale alors le franchissement d'un seuil moral qui départage le bon accompagnement de celui qui, trop pressant ou trop soucieux, menace de se transformer en mal infligé en éloignant de l'émancipation attendue. C'est dans la considération de ce seuil même que la responsable du dispositif marque les limites dans lesquelles elle estime que l'accompagnement doit impérativement rester.

"Il faut bien savoir jusqu'où aller. C'est la question qui avait été posé lors de la réunion [réunissant l'ensemble des parrains et les responsables du dispositif pour évaluer les actions en cours]: est-ce que le parrain accompagnait aux entretiens d'embauche, c'est-à-dire concrètement, son filleul ? Et là on avait dit non. Parce que si il l'accompagne concrètement pour dire " eh bien voilà mon filleul» et être là à l'entretien d'embauche, eh bien l'entreprise va dire " il manque d'autonomie »».

À ce niveau de reconnaissance où s'atteste la consistance d'une volonté de l'usager à maintenir un cheminement vers l'insertion, le Conseiller Emploi veille également à sa capacité à s'instruire des défaveurs rencontrées dans les diverses épreuves traversées. Par là, le jeune doit se maintenir, sans se défaire, dans une dynamique d'apprentissage qui accepte la sanction et la considère justifiée ${ }^{10}$. Lorsque les différents éléments qui attestent de la consistance font défaut, les travailleurs sociaux tardent à le laisser aller sans soutien aux épreuves du marché du travail. Il arrive néanmoins qu'ils y précipitent quelques usagers : lorsque leurs conseils ne portent pas, plutôt que de risquer, en insistant, de s'aliéner leur confiance, ils s'en remettent alors à la simple sanction des épreuves de recrutement. Dans de tels cas, il s'agit pour eux de faire en sorte que le jeune s'instruise de l'issue imposée par le marché du travail. Et si cela s'avère, l'apprentissage permis par le travail d'accompagnement aura porté ses fruits : l'usager n'aura pas décroché de son parcours d'insertion et il se sera maintenu face aux exigences conventionnelles situées au seuil du recrutement.

La persévérance dans l'exposition aux épreuves du recrutement participent de l'effort considérable demandé aux usagers qui veulent s'insérer par le biais des Missions Locales. On a vu combien l'exigence capacitaire demandées par ces épreuves réclame l'assurance d'une préparation des usagers. C'est un travail de la proximité, au cœur de l'accompagnement

\footnotetext{
${ }^{10}$ Ce qui ne va pas sans problèmes lorsque les épreuves sont infectées par des discriminations illégitimes.
} 
social, qui s'y consacre, au prix d'un passage sous les exigences conventionnelles et au bénéfice d'un apprentissage touchant son but dans la reconnaissance d'un usager moralement responsable et capable de soutenir des projets autonomes [Pattaroni, 2005].

\section{CONCLUSION : LA VIGILANCE CRITIQUE AUTORISÉE PAR LE « DOUBLE PLURALISME »}

L'attention portée à des engagements non conventionnels nous semble favoriser la vigilance critique du sociologue et la placer sous l'égide d'une politique du double pluralisme [Breviglieri \& Stavo-Debauge, 1999]. Un double pluralisme suppose une attention simultanée au niveau horizontal des conventions de jugement et au niveau vertical des modalités d'engagement en commun qui vont du lien entre les proches jusqu'aux rapports en public. Une politique du double pluralisme doit alors conduire une double visée : non seulement assurer un sens du juste en maintenant ouvert l'accès à la diversité des biens communs et en garantissant que les principes de justice puissent faire l'objet de choix et d'associations ; mais aussi en préservant une considération pour des modalités d'engagement dans le monde qui ne passent pas par un sens du juste et qui laissent advenir des biens dont la portée est d'extension variable. La vigilance autorisée par une politique du double pluralisme est d'autant plus aiguë qu'aux lieux multiples d'injustice qu'elle peut identifier (lorsqu'un principe de justice fait défaut ou inversement domine fortement ou radicalement les autres) s'ajoutent des sources d'humiliation et de souffrance (lorsqu'un régime d'engagement est déconsidéré au profit d'autres). Le spectre des modèles de tyrannie et des formes d'oppression dont il faut se départir s'en trouve d'autant étendu.

Le travail de la proximité visant à accompagner des personnes dans un parcours d'insertion et à les apprêter aux épreuves de recrutement nous permet d'envisager pleinement ce point politique. Déconsidérer les attentes de proximité et de sollicitude des personnes particulièrement vulnérables et les confronter trop directement aux attentes conventionnelles dont regorgent les épreuves professionnelles consiste à les exposer trop hâtivement à la brutalité probable d'une sanction publique dévalorisante. Le travail de la proximité qui entend parer à ces formes diverses et particulièrement profondes de vulnérabilité trouve ainsi un mérite digne d'estime sociale et de reconnaissance professionnelle. Du même coup, une seconde source d'humiliation réside dans le déni de reconnaissance des compétences de proximité que ces travailleurs sociaux développent dans l'exercice de leur métier [Breviglieri, 2005].

Ce travail de la proximité en direction des usagers ne saurait pourtant suffire, et cela spécialement pour une action publique qui est censée participer d'une lutte contre les discriminations. Pour bien faire, l'entreprise consistant à apprêter les usagers aux exigences des épreuves se devrait d'être symétrisée en portant pareillement sur lesdites épreuves auxquelles ceux-ci sont destinés à prendre part. La politique ici conduite devrait ainsi avoir son pendant pour ce qui concerne l'environnement d'épreuves dont sont passibles les personnes. Car l'environnement, tout comme les épreuves, réclame d'être adéquatement apprêté afin de pouvoir donner place et de faire droit à la variété des engagements des personnes ainsi qu'aux biens et principes qui importent à la communauté [Stavo-Debauge, 2005].

Or, les dispositifs des Missions Locales se trouvent être, sur ce point, sujets à de légitimes critiques. Certes, le Parrainage à l'emploi veille à apprêter les jeunes aux attentes des épreuves de recrutement et, plus largement, aux exigences du monde du travail. Mais si 
les parrains et les travailleurs sociaux s'arrêtent au seuil des épreuves de recrutement proprement dites, afin de pas attenter à l'autonomisation de leur public, force est de constater que cet arrêt vaut également pour ce qui concerne l'inspection et l'encadrement des épreuves contre lesquels ce même public ne cesse pourtant de buter. Alors que les autorités publiques ont appréhendé le parrainage à l'emploi comme un dispositif de lutte contre les discriminations, c'est au seuil même des lieux où les discriminations s'exercent que l'action publique coupe son effort de vigilance. Le parrainage ne s'inscrit dans aucun plan d'action s'armant de la force du droit et soutenant une scrutation des épreuves de recrutement et une révision des conventions qu'elles embarquent.

\section{BIBLIOGRAPHIE}

BAIER, A. (1986), « Trust and anti-trust », Ethics, 96(2).

Benlemselmi, A. (2002), Analyse de parcours d'insertion de jeunes filles issues de l'immigration au travers leurs récits de vie, Mémoire de DEA d'Économie des Institutions, Université de Paris X-Nanterre.

Boltanski, L. (1990), L’amour et la justice comme compétences, Métaillié.

BoltANSKI, L. \& ThÉVENOT, L. (1991), De la justification, Gallimard.

Boltanski, L. \& Chiapello, E. (1999), Le nouvel esprit du capitalisme, Gallimard.

Bessy, C. \& EyMARD-DuvernAy, F. (1995), Les intermédiaires du marché du travail, CEEPUF.

Breviglieri, M. (2005), « Bienfaits et méfaits de la proximité dans le travail social », in ION, J. (dir.), Le travail social en débat[s], Éd. La Découverte.

Breviglieri M. \& Stavo-Debauge J. (1999), «Le geste pragmatique de la sociologie française », Antropolitica, 7.

Breviglieri, M., Pattaroni, L. \& Stavo-Debauge, J. (2003), « Quelques effets de l'idée de proximité sur la conduite et le devenir du travail social», Revue suisse de sociologie,29, (1).

Dodier, N. (1991), « Agir dans plusieurs mondes », Critique, 529-530.

Eymard-Duvernay, F., MARChal, E. \& Alii. (1997), Façons de recruter, Métailié.

GoffMAn, E. (1973), La mise en scène de la vie quotidienne. 2 vol., Éd. de Minuit.

JosePH, I. (1998), Erving Goffman et la microsociologie, PUF, 1998.

LIVET, P. (1993), «Théorie de l'action et conventions », in LADRIÈRE, P. \& ALii. (eds), La théorie de l'action. Le sujet pratique en débat, Ed. CNRS.

Pattaroni, P. (2005), Politiques de la responsabilité. Promesses et limites d'un monde fondé sur l'autonomie, Thèse de doctorat, EHESS et Université de Genève.

QUÉRÉ, L. (1993), « À-t-on vraiment besoin de la notion de convention ? », Réseaux, 62.

RiCEUR, P. (2001), Le juste II, Esprit.

Ricelur, P. (2004), Parcours de la reconnaissance, Plon. 
Stavo-Debauge, J. (2005, à par.), «Aux seuils de «l'intégration» et en butte aux « discriminations ». Apprêter et soutenir une volonté, Le Parrainage à l'emploi » in L. ThÉVEnot (dir.) Politiques au regard du proche.

ThÉvenot, L. (1994), « Le régime de familiarité. Des choses en personnes », Genèses, 17.

ThÉVEnot, L. (2005), «Biens et réalités de la vie en société. Dispositions et compositions d'engagements pluriels » in Breviglieri, M., Lafaye, C. \& Trom, D. (eds), Sens de la justice, sens de la critique, Économica. 